\title{
An Ellipsoidal 3D Shape Representation and Wavelet Transform Feature Descriptor for 3D Shape Retrieval
}

\author{
*Asma Khatun \\ Faculty of Computer Science and Information \\ Technology \\ Universiti Malaysia Sarawak \\ kasma@pgfit.unimas.my
}

\author{
Prof. Wang Yin Chai \\ Faculty of Computer Science and Information \\ Technology \\ Universiti Malaysia Sarawak \\ Sarawak, Malaysia
}

\author{
Md. Rabiul Islam \\ Faculty of Computer Science and Information Technology \\ Universiti Malaysia Sarawak \\ Sarawak, Malaysia
}

\begin{abstract}
For the year of past most three-dimensional objects are represented and mapped with spherical function. Since the mapping preserves important criteria such as geometrical information of a 3D object, the representation of a 3D shape in a suitable way is an important matter for $3 D$ object retrieval. In many circumstances, spherical surface fitting to a 3D shape does not approximate very well, because of their limited degree of divergence by their radius only. Whereas, in the case of ellipsoid definition we get three axes, which gives us a free mapping along each of $x, y$ and $z$ axes. Due to higher degree of freedom ellipsoid give better approximation for a given $3 D$ shape than sphere which lead to a less error and higher discriminant shape descriptor for retrieval of $3 D$ shape. In this paper, we describe a new 3D shape representation method based on ellipsoid for the purpose of 3D shape retrieval. And we demonstrate our experimental result tested on standard 3D shape Princeton Shape Benchmark (PSB) database, inferring new wavelet shape descriptor of the represented 3D shape function on ellipsoid. Our experimental results support our expectation of our proposed hypothesis.
\end{abstract}

Keywords- Shape Representation; Ellipsoid Approximation; Ellipsoidal Wavelet; 3D Shape Retrieval; Wavelet Descriptor; Computer Vision

\section{INTRODUCTION}

3D shape retrieval continues to be one of the most exciting and fastest growing research areas in the field of computer vision, molecular biology, computer graphics, $\mathrm{CAD} / \mathrm{CAM}$ engineering system, car industry, entertainment even in digital arts and a variety of other fields. A challenge is to find a suitable 3D shape retrieval technique, with high and unique discrimination between similar and dissimilar shapes. Shape representation technique is an important issue for the purpose of retrieving the 3D shape since it stores important information of the 3D shape.

However, existing research are mostly based on function representing on sphere for the purpose of 3D shape retrieval. Vranic et al. [1, 2], Novotni et al. [3] Kazhdan et al. [4] and Mousa et al. [5] are the examples of 3D shape descriptor that has been used for the 3D shape representation. Their aim was to estimate feature vector of spherical harmonic coefficient of a 3D triangular mesh model representing shape function on sphere. There is also another shape feature vector of spherical wavelet coefficients Laga et al. [6], representing 3D shape function on sphere. However, Feature vector approaches are more effective due to higher discrimination. Still we found that sampling is irregular for spherical harmonics, which demonstrated details by Laga et al. [6]. Additionally, spherical shape functions have poor control of coefficient due to higher divergence and lower degree of freedom. And the spherical shape approximation is not closer to the $3 \mathrm{D}$ object which contributes more error prone and less accurate shape descriptor.

There is also another work in this research area. It compares graphs for recognition of 3D shape retrieval, or represents histogram of shape variation or shape distribution. Directional Histogram Model (DHM) proposed by Liu et al. [7] represented histogram of thickness distribution of two points on the object surface. Another approach Osada et al. [8] represented histogram of shape distribution of some geometric property such as D2, which represents distance between two random points on the surface. These methods are easy to compute but are less efficient with respect to their discriminative ability. Skeletal graph by Sundar et al. [9] stored the skeletal connected node information to compare 
resultant graph and Multiresolution Reeb Graphs (MRGs) by Hilaga et al. [10] are most common technique used for measuring shape similarity comparing as a graph. Another Sketelon, defined by Reeb [11] formed by continuous scalar function used as height function, distribution of geodesic distance on the object. However, these graphical methods are more suitable for the shapes which are segmented joint but these schemes are usually much more computationally expensive and thus not suitable for real application. Additionally, Reeb graph is not robust in respect to feature extraction performance. Large graph size decrease efficiency. Another shape measurement method proposed by Lian et al. [12] evaluating rectilinearity (proportionally to the ratio of the sum of three orthogonal projected areas and the surface area of the mesh). The property of this measure was estimated rectilinearity is always from 0 to 1 . Whether it performs better as compared with PCA in some cases but measurement is not optimal as example for cube, result is not exactly 1 , which leads to poor discrimination.

The main goal of the research is to find a suitable 3D shape representation scheme and to estimate high discriminating and less error prone shape descriptor for 3D shape retrieval. Our main consideration of shape representation method is to preserve information as example geometrical information of a 3D shape model as much as possible for a better shape descriptor. Mostly existing shape representation techniques are related to representing 3D shape function on a sphere. However in some circumstances spherical functions are not closest fitting to the shape of the body. Our proposed shape representation method based on ellipsoid. The advantage is that, ellipsoid gives more closure approximation and high degree freedom of a given 3D shape rather than sphere. Here, we have proposed a new ellipsoidal wavelet descriptor representing shape function based on ellipsoid of a 3D shape model. Our approach represents 3D shape model (triangular model) on the ellipsoid to approximate and mapped into a square image for further step. Finally, an ellipsoidal wavelet transform is computed to obtain the shape descriptor of wavelet coefficient. As a result we get more high discrimination and accurate feature vector of ellipsoidal shape function since our approximation method gives more controlling of coefficients.

\section{3D SHAPE REPRESENTATION}

We use ellipsoid to approximate the 3D shape model in our proposed shape representation method. The reason of choosing the ellipsoid is as follows:

- In general 3D shapes are irregular in nature. All existing methods are suitable for near spherical bodies not for nonspherical bodies which create problem to compute expansion close to the surface of the bodies. The approximation of a 3D shape with a sphere exposed to be intensely high, Fig. 1 shows the smallest sphere enclosing the whole body. But for the case of ellipsoid, it can be easily shown that the uniform fitting approximate more closure to the body of the shape of the given 3D shape in Fig. 2.
- Moreover ellipsoidal mapping surface don't have any pole like spherical mapping surface which have singularity problem to the North and South Pole which affect the sampling.

- Spherical mapping used for texture mapping often does not fit in the surface very well because they have limited degree of freedom within which they are approximated.

- One notable benefit is that, ellipsoids are defined by three axes, one along each of the $\mathrm{x}, \mathrm{y}$ and $\mathrm{z}$ axis and this is why ellipsoids gives more accurate representation to the body of the shape. Whereas a sphere only altered by its radius, Fig. 3 shows the different ellipsoid of these three axis definition.

- Forming multilevel resolution Wavelet based descriptor is more suitable on image. The ellipsoidal model has advantage to construct large-scale detail since texture mapping is also affected by the way of approximation to the shape model.

- And obviously the computation error will be lower as well as higher accuracy in case of ellipsoid due to aforementioned advantages of ellipsoidal shape function.

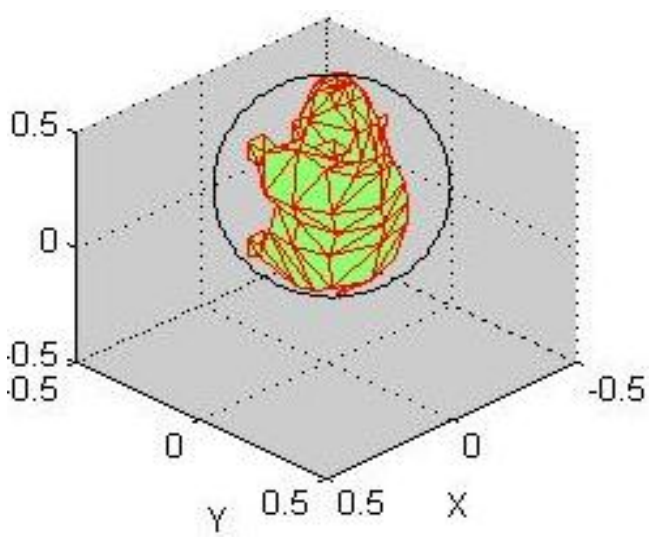

Figure 1. A 3D shape model enclosed with sphere 


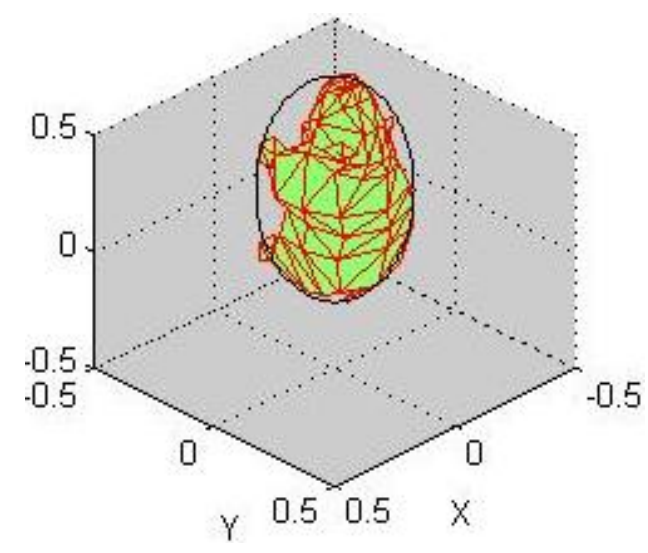

Figure 2. A 3D shape model enclosed with ellipsoid

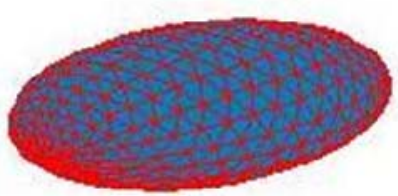

$y>x>z>0$

(a)

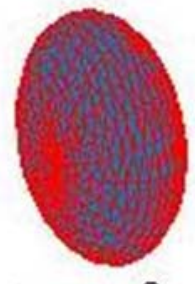

$z>x>y>0$

(c)

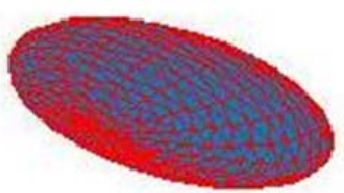

$x>y>z>0$

(b)

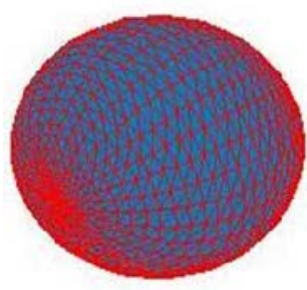

(d)
Figure 3. Different ellipsoid (a, b, c) along their axis and sphere (d) with radius $r$

\section{PROPOSED METHOD}

Our proposed method consists of two steps and is describing follows. The first step is the process of shape representation method approximated by ellipsoid of a 3D triangular model. Second step describes shape recognition process which extract wavelet coefficient for estimating unique feature vector. And ultimate we get a new shape descriptor of a 3D shape function based on ellipsoid. To measure the similarity of two shape descriptor we use Euclidian norm. The whole procedure of the proposed method has been detailed following.

\section{A. 3D Object Representation}

Representing 3D shape is an essential stage and important goal to preserve the important chracteristics for the purpose of $3 \mathrm{D}$ recognition purpose in computer vision. As it was earliar mentioned that the major problem to estimate high discrimintive shape information. Here we present a new geometric descriptor representing shape function on ellipsoid. Already we mentioned the reason behind using ellipsoid as a primitive. The another contributions of our proposed shape representation are the following.

- The proposed shape representation method can be use represented as surface and also solid or volume approximation.

- The ellipsoid represented surface can be easily converted to other shape such as sphere.

Ellipsoidal mapping of $3 D$ object: We estimate the ellipsoidal parameterized approximation by mapping the $3 \mathrm{D}$ triangular shape model radially from the shape to the vertices of the unit ellipsoid centre. The ellipsoid mapping is defined by the equation

$$
\frac{X_{1}^{2}}{a^{2}}+\frac{Y_{1}^{2}}{b^{2}}+\frac{Z_{1}^{2}}{c^{2}}=1
$$

where semi axes $a, b, c \neq 0$.

And the sampling vertices are defined to ellipsoid as

$$
\begin{aligned}
& X_{1}=f_{v}\left(\mathrm{a}^{*} \mathrm{x}+x_{c}\right) \\
& Y_{1}=f_{v}\left(\mathrm{~b}^{*} \mathrm{y}+y_{c}\right) \\
& Z_{1}=f_{z}\left(\mathrm{c}^{*} \mathrm{z}+z_{c}\right) .
\end{aligned}
$$

Where $\left(\mathrm{X}_{1}, \mathrm{Y}_{1}, \mathrm{Z}_{1}\right)$ is ellipsoidal mapped parameter and $\mathrm{f}_{\mathrm{v}}$ define the sampled vertices with radii $\mathrm{a}, \mathrm{b}$ and $\mathrm{c}$ and the centre $\left(\mathrm{x}_{\mathrm{c}}, \mathrm{y}_{\mathrm{c}}, \mathrm{z}_{\mathrm{c}}\right)$ and $\mathrm{x}, \mathrm{y}, \mathrm{z}$ are the spherical coordinate with $\theta\{0, \pi\}$ and $\varphi\{0,2 \pi\}$. Since the ellipsoid sampling is more uniform to the shape than sphere, our proposed approach avoids the practical limitation of measuring similarity of the same object in different polygon connectivity.

As a preprocessing technique we applied Principal Component Analysis (PCA), Jollife [13] on a 3D model on each of its vertices. This method consists of set of transformation such as translation, rotation and scaling.

\section{B. Shape Recognition}

Ellipsoid to planar map: It is suitable to estimate wavelet coefficient on the two-dimensional image. So, we simply map the approximated shape function to a square image I with size $\mathrm{m} \times \mathrm{m}$ (for our case $\mathrm{m}$ is 64) taking into account that each point on the surface map to a unique point on the texture square. Here also we benefited that,

- Ellipsoidal projection mapping does not have any pole like spherical projection map and for spherical the small areas around the poles map in a highly distorted manner all over the top and bottom of the square.

Wavelet Transform: The wavelet was introduced by Morlet and Grossman (1984) as a time-scale analysis tool for non- 
stationary signals. It was further developed by many authors (e.g. Mallat, 1989: Daubechies, 1990) and rapidly found application in many areas. A wavelet function is a function that is well localized in the space and frequency domains. Nowadays wavelet basis are becoming the foundation for the most popular techniques for signal processing and image processing in a wide range of applications.

We applied 3-level Haar wavelet transform on the image I in order to generate unique characteristics vector. A twodimensional image signal is wavelet-transformed in horizontal and vertical directions, and the image is divided into four regions ' $\mathrm{LL}$ ', 'HL', 'LH' and ' $\mathrm{HH}$ ' after the wavelet transform has been performed once, as shown in fig. 4. The 'LL' region contains only low frequency components (the approximation function component) and ' $\mathrm{HH}$ ' region contains high frequency component. Once we have performed a 1-level Haar transform, then it is easy to repeat the process and perform multiple level transform. The first level Haar transform of an image $f$ is defined by

$$
f \rightarrow\left(\frac{h^{1}}{a^{1}} \frac{d^{1}}{v^{1}}\right) .
$$

Where $h^{1}, v^{1}, d^{1}$ is the first horizontal, vertical and diagonal fluctuation respectively and $a^{1}$ is the approximation image.

\begin{tabular}{|c|c|}
\hline LL & LH \\
\hline HL & HH \\
\hline
\end{tabular}

Figure 4. 1-level 2D wavelet decomposition

Descriptor extraction: We estimated feature vector collecting the wavelet detail coefficient, $\left\{\mathrm{h}^{0} \ldots \mathrm{h}^{\mathrm{n}-1}\right\},\left\{\mathrm{v}^{0} \ldots \mathrm{v}^{\mathrm{n}-1}\right\},\left\{\mathrm{d}^{0} \ldots \mathrm{d}^{\mathrm{n}-}\right.$ $\left.{ }^{1}\right\}$ up to level $\mathrm{n}$. We processed the value as a binary value for making feature vector. It is expensive to work with all coefficients, so we recommend taking first 256 data coefficient to estimate feature vector.

\section{Similarity Measure and Error Estimation}

If $F_{1}$ and $F_{2}$ are two feature vectors of two $3 \mathrm{D}$ objects $\mathrm{O}_{1}$ and $\mathrm{O}_{2}$, the distance between two descriptor of $\mathrm{L}_{2}$ norm is:

$$
D\left(F_{1}, F_{2}\right)=\sqrt{\sum_{i}\left(F_{1}-F_{2}\right)^{2}} .
$$

We measure the approximation error by the distance between the surface $\mathrm{X}$ and ellipsoid $\mathrm{O}$ as

$$
E_{\text {sur }}(O)=\max \left\{\left\|x_{v}-v\left(P_{o}\right)\right\|, x_{v} C X\right\} .
$$

where $v\left(P_{o}\right)$ means to radial projection of vertices on ellipsoid O.

\section{RESULTS AND DISCUSSION}

To evaluate the accuracy of proposed approach extensive experiments on well known database PSB [17] are performed. We used a standard database Princeton Shape Benchmark (PSB) in 3D (OFF) file format. We measured the approximation error of the 3D models representing function on ellipsoid. We compared the approximation error of our method with spherical representation function. The comparison of approximation error is with two examples dog and human is shown in Fig. 5(a) and with shark and tree in Fig. 5(b). Some of the tested models are shown in Fig. 6.

In order to evaluate the measurement of retrieval performance we examine precision/recall diagram for the shape descriptor of 3D objects. Briefly, recall is the proportion of the relevant models actually retrieved and precision is proportion of retrieved models that is relevant. And we shown the comparison of the retrieval performance between spherical based wavelet shape descriptor and our proposed ellipsoid based shape descriptor in Fig. 7. Another precision/recall diagram (Fig. 8) compared for the fish and shark model tested from the Princeton Shape Benchmark database (PSB).

From our experiment we can demonstrate that our proposed approach reduce approximation error definitely. For example the human model, error falls from $60 \%$ from $30 \%$. This provides greater accuracy for estimating shape descriptor for similarity measure.

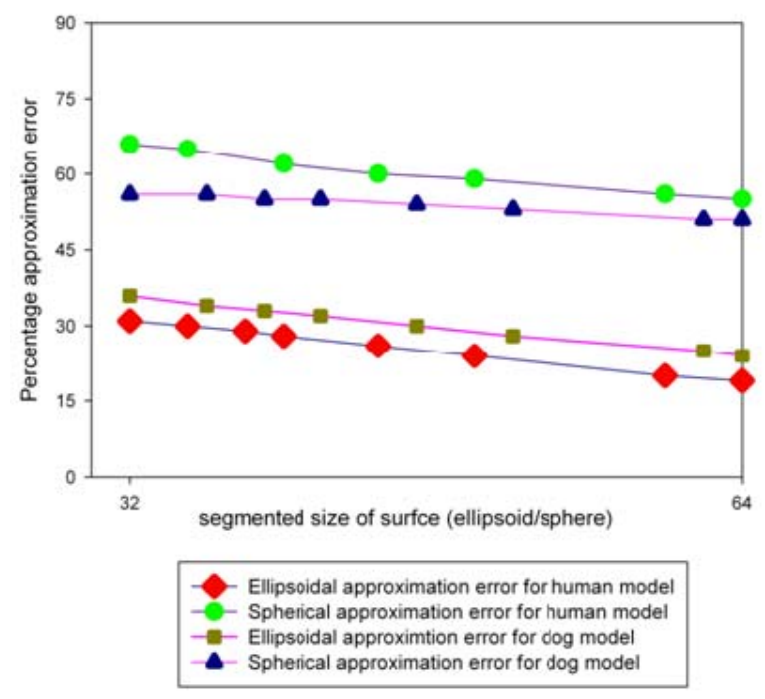




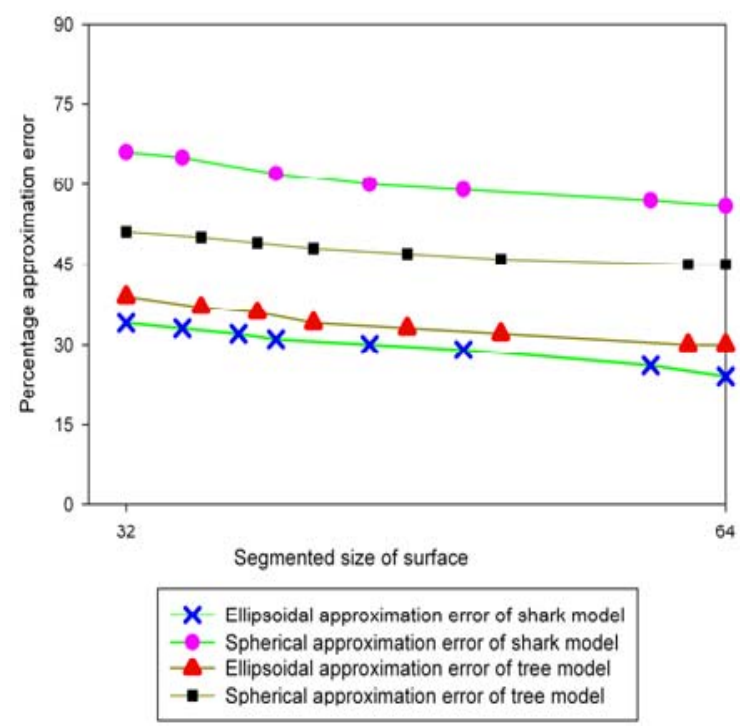

(b)

Figure 5(a). Comparison of approximation error for dog and human model, (b) Comparison of approximation error for shark and tree model

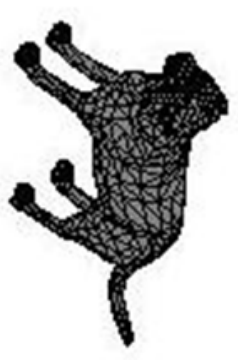

(a) $\operatorname{dog}$

(c) shark

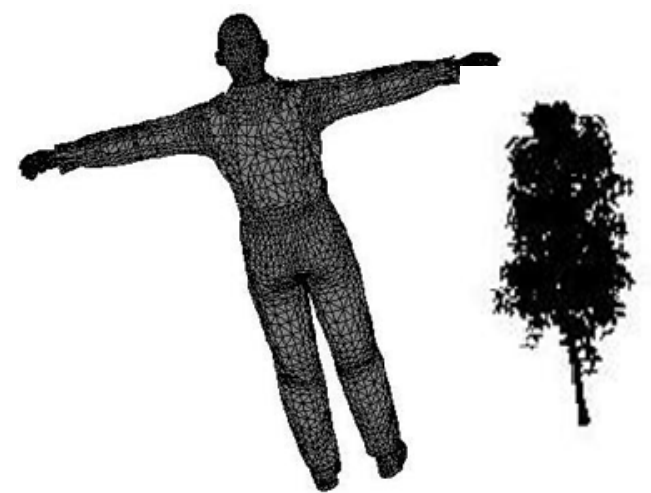

(b) human

(d) tree

Figure 6.Some of models used for testing our approach

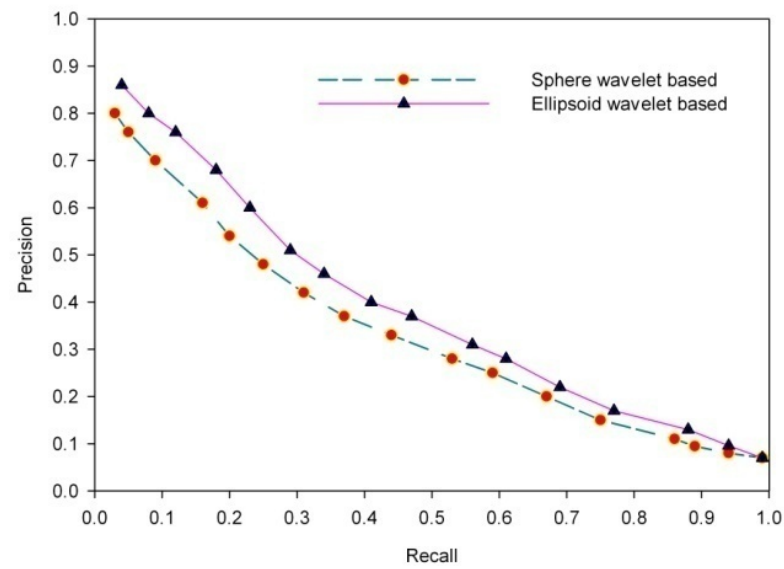

Figure 7.Precision/recall diagram for 3D models of PSB database 


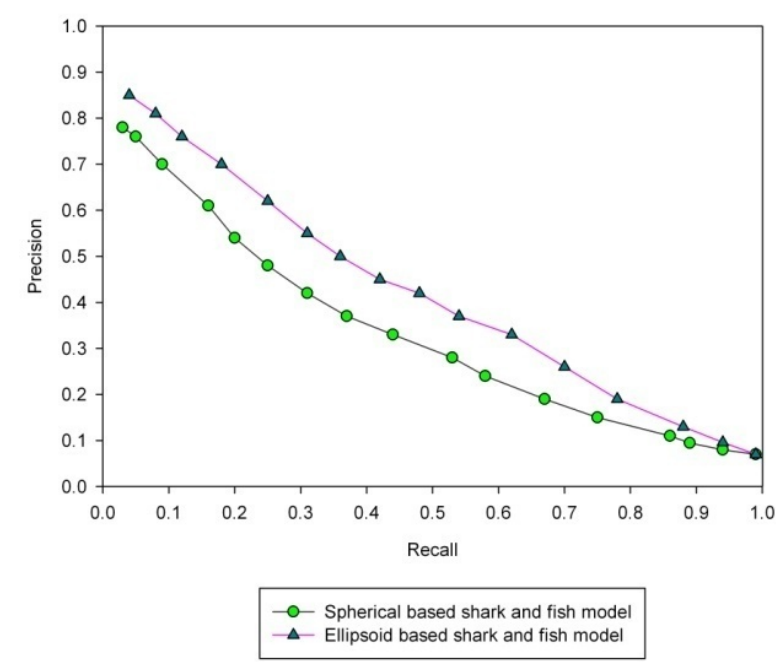

Figure 8. Precision/recall diagram for fish and shark model

\section{CONCLUSION AND FUTURE WORKS}

A 3D model retrieval based on ellipsoidal wavelet is proposed. We approximate 3D model on ellipsoid in order to extract feature vector for any 3D model using wavelet transform. Our proposed descriptor is compared to existing descriptor based on spherical function with respect to the accuracy and approximation error. Our approach shows greature accurcy in terms of retrieval performance.

There are some factors to consider incase of approximation of $3 \mathrm{D}$ object with any function such as sphere or ellipsoid or any other:

- Approximation should be very close to the body of the shape.

- It should cover the whole body with detail joint part for example, a human body with his hand or a dog with his legs.

In our proposed approch we satisfy with first factor. But in contrary, it is very difficult to cover exactly the 3D shape which have complex joint part with a closest fitting by our proposed shape representation sceme.

So our further work is continuing considering with the above mentioned limitation. A possible way to solve this problem is to represent a 3D shape in a tree structure or graph structure to cover the detail joint part. So our next aim is to find any possibility to produce hybridization method with our method. One possible way is to create a tree structure of the 3D shape with a no. of node and to approximate with ellipsoid. But the problem is that it will be very complex which is very impossible to apply in a real application since may require many hundreds of node. We are considering this situation for future work.

\section{REFERENCES}

[1] V. Vranic and D. Saupe, "Description of 3D- shape using a complex function on the sphere," Proceedings of IEEE International Conference on Multimedia and Expo (ICME), pp: $177-180,2002$.

[2] V. Vranic, "An improvement of rotation invariant 3D shape descriptor based on functions on concentric spheres," Proceedings of IEEE International Conference on Image Processing (ICIP), pp: 757-760, 2003.

[3] M. Novotni and R. Klein, "3D Zernike descriptors for content based shape retrieval," Proceedings of the eighth ACM Symposium on Solid Modeling and Applications, pp: 216-225, 2003.

[4] M. Kazhdan, T. Funkhouser and S. Rusinkiewicz, "Rotation invariant spherical harmonic representation of $3 \mathrm{D}$ shape descriptors," Proceedings of the 2003 Eurographics/ACM Symposium on Geometry Processing, pp: 156-164, 2003.

[5] M. Mousa, R. Chaine, S. Akkouche and E. Galin, "Efficient spherical harmonics representation of 3D objects," Proceedings of the 15th Pacific Conference on Computer Graphics and Applications, pp: 248-255, 2007.

[6] H. Laga, M. Nakajima and K. Chihara, "Discriminative spherical wavelet features for content-based 3D model retrieval," International Journal of Shape Modeling, pp: 51-72, 2007.

[7] X. Liu, R. Sun, S. B. Kang and H. Y. Shum, "Directional histogram model for three-dimensional shape similarity," Proceedings of the IEEE Computer Society Conference on Computer Vision and Pattern Recognition (CVPR), pp: 813-820, 2003.

[8] R. Osada, T. Funkhouser, B. Chazelle and D. Dobkin, "Shape distributions," ACM Transactions on Graphics (TOG), pp: 807-32, 2002.

[9] H. Sundar, Silver, N. Gagvani and S. Dickinson, "Skeleton based shape matching and retrieval," International Conference on Shape Modeling and Applications, pp: 130-139, 2003.

[10] M. Hilaga, Y. Shinagawa, T. Kohmura and T. L. Kunii, "Topology matching for fully automatic similarity estimation of 3D shapes," Proceedings of the 28th Annual Conference on Computer Graphics and Interactive Techniques (SIGGRAPH), pp: 203-212, 2001.

[11] Reeb, "On the singular points of a completely integrable pfaff form or of a numerical function," Comptes Rendus Acad. Sciences Paris, pp: 847-849, 1946.

[12] Z. Lian Z, P. L. Rosin and X. Sun, "A rectilinearity measurement for 3D meshes," Proceedings of the $1^{\text {st }}$ ACM International Conference on Multimedia Information Retrieval, Vancouver, British Columbia, Canada, pp: 395-402, 2008.

[13] Jolliffe, "Principal component analysis," Springer-Verlag, 1986. 